\title{
THE HIGH RESOLUTION SPECTRUM OF THE PULSATING, PRE-WHITE DWARF STAR PG 1159-035 (GW VIR) (1)
}

\author{
James Liebert (2) \\ Steward Observatory, University of Arizona \\ AZ 85721
}

F. Wesemael (2)

Departement de Physique and Observatoire du Mont Megantic, Université de Montréal, C.P. 6128, Succ. A, Montréal, PQ, H3C 3J7, Canada

D. Husfeld

Institut für Astronomie und Astrophysik der Universität München, Scheinerstrasse 1

D-8000 München 80

R. Wehrse (2)

Institut für Theoretische Astrophysik der

Universität Heidelberg, D-6900 Heidelberg, F.R. Germany

S. G. Starrfield

Department of Physics, Arizona State University

Tempe, AZ 85287

and

E. M. Sion

Department of Astronomy, Villanova University

Villanova, PA 19085

\section{INTRODUCTION}

First reported at the IAU Colloquium No. 53 on White Dwarfs (McGraw et al. 1979), PG 1159-035 (GW Vir) is the prototype of a new class of very hot, pulsating, pre-white dwarf stars. It shows complicated, nonradial pulsation modes which have been studied exhaustively, both observationally and theoretically. The effective temperature has been crudely estimated as $100,000 \mathrm{~K}$ with $\log g \sim 7$ (Wesemael, Green and Liebert 1985, hereafter WGL).

(1) Optical data reported here were obtained at the Multiple Mirror Telescope (MMT) Observatory, a facility operated jointly by the Harvard-Smithsonian Center for Astrophysics and the University of Arizona.

(2) Guest Observers with the International Ultraviolet Explorer (IUE) Observatory, which is sponsored and operated by the National Aeronautics and Space Administration, the European Space Agency and the Science Research Council of the United Kingdom. 
The optical spectrum shows broad absorption features attributable primarily to He II, C IV and O VI, often with central emission reversals; no hydrogen lines are seen. The abundances in the atmosphere are of great interest with respect to the pulsational driving mechanism. The preliminary analysis of WGL assumed that the star had a composition similar to the hot DO white dwarfs, with helium the dominant constituent. However, Starrfield et al. (1984) predicted that the pulsational driving required very high abundances of oxygen and/or carbon near the surface; too much helium would quench the pulsations. This prediction led directly to the discovery of the O VI features at optical wavelengths (Sion, Liebert and Starrfield 1985). However, the ultraviolet wavelengths accessible to the International Ultraviolet Explorer (IUE) Observatory are of greater importance in the identification and analysis of spectroscopic features due to CNO ions and potentially those of any heavier elements. In this paper we report results from a long IUE echelle observation covering the short wavelength (SWP) bandpass. Additional low resolution IUE data and a high resolution observation of the optical $4686 \mathrm{~A}$ region were also obtained.

\section{THE OBSERVATIONS}

Contiguous European and US1 shifts, were used to obtain a 17 hour SWP echelle exposure on 1984 May 16-17. The spectrum showed a rich spectrum of absorption lines. Identified transitions, velocities and estimated equivalent widths are listed in a forthcoming paper by the same authors. We summarize the principal findings below.

Lines showing cores distinct enough for velocity estimates included $\mathrm{N} \mathrm{V} \mathrm{1238,} \mathrm{1242A} \mathrm{(Fig.}$ 1), N IV 1270A, O V 1371A, and C IV 1548, 1551A (Fig. 2). The Si IV 1394, 1402A doublet is not seen. There is little evidence for fast or slow wind components in any resonance lines. C IV shows strong, broad absorption at both doublet reversals, with possible evidence for central emission reversals. The C IV values $(+13,+24 \mathrm{~km} / \mathrm{sec})$ are marginally consistent with each other, but significantly lower than the average found from the other lines $(+35 \mathrm{~km} / \mathrm{sec})$. Since the C IV cores are poorly defined, we accept the latter as the most likely photospheric velocity. A consistent set of low excitation lines at a velocity of $-28 \mathrm{~km} / \mathrm{sec}$ is attributed to an interstellar cloud.

One very broad feature, with an equivalent width of nearly $2 \mathrm{~A}$ and spanning 1341-55 A (Fig. 3), is shallow and complicated compared with the other strong lines. This feature had been noted in low dispersion IUE spectra of PG 1159-035 and similar stars (WGL, Bond et al. 1984), but its wavelength remained ambiguous. The new spectrum shows that our earlier suggested identifications with C III and N III are incorrect. We have found that this and other broad features noted in the high dispersion image may be identified with transitions of highly-excited levels of C IV, calculated by one of us (D.H.) in analyzing the spectrum of the central star of the planetary nebula NGC 246 (Husfeld 1987).

An optical spectrum was obtained with the Multiple Mirror Telescope on 1988 January 2 showing the emission reversal at the center of the broad 4686A absorption (see WGL). The stellar radial velocity, as measured by cross-correlation of the central feature with a Gaussian emission 


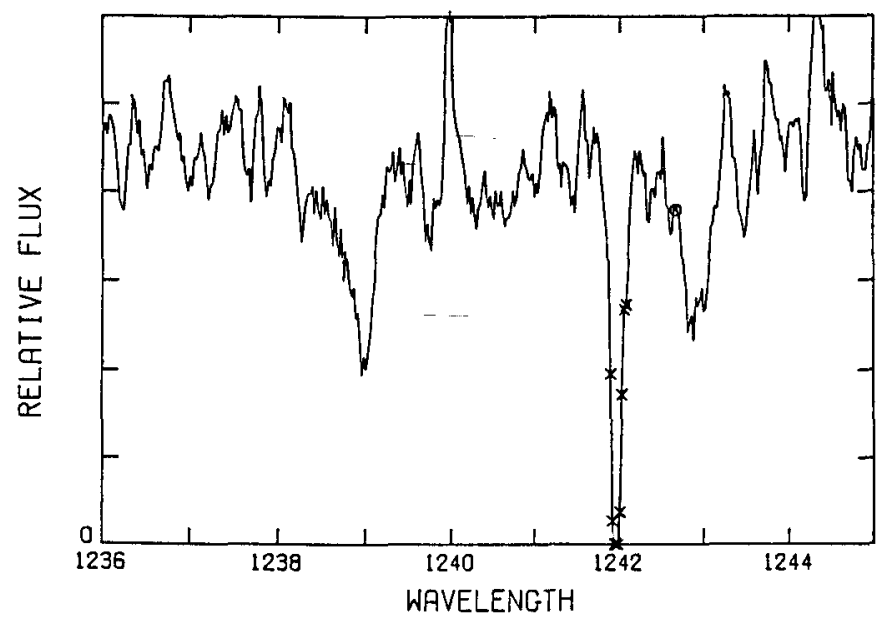

Figure 1. A detailed plot of the region of the N V 1238.82, 1242.80A doublet in the high dispersion spectrum.

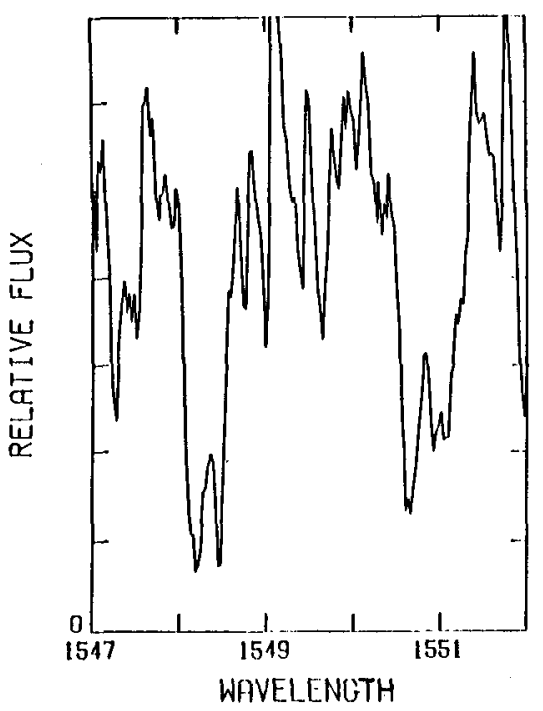

Figure 2. A similar plot showing the C IV $1548.18,1550.77 \mathrm{~A}$ doublet.

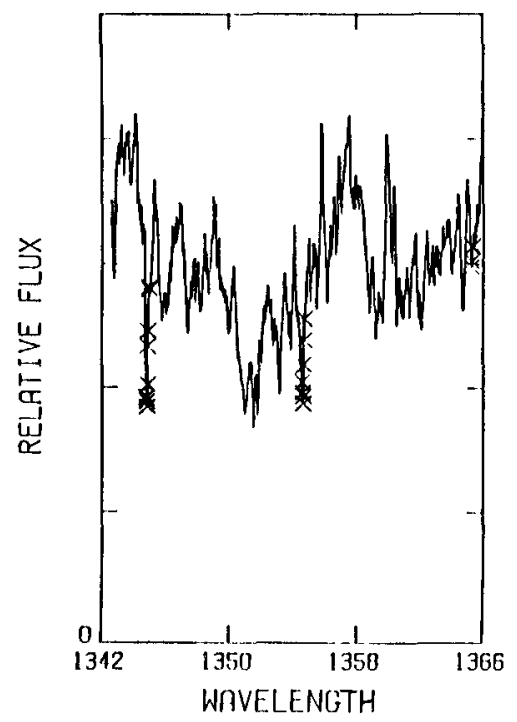

Figure 3. A plot of the likely C IV absorption (and possibly other) features in the $1346-54 \mathrm{~A}$ region of the high dispersion spectrum which has a total measured EW of nearly $2 \mathrm{~A}$. Identified transitions include lines at $1351.2 \mathrm{~A}, 1352.9$ and $1358.6 \mathrm{~A}$. 
line profile is $+48.4 \pm 2.9 \mathrm{~km} / \mathrm{sec}$ assuming identification with $\mathrm{He}$ II, and $+80 \mathrm{~km} / \mathrm{sec}$ assuming C IV. However, recent calculations of Kudritzki (1987) applicable to helium-rich planetary nebula nuclei and hot subdwarfs predict such a feature will be caused by ongoing mass loss, and may show a velocity shift with respect to the photosphere.

\section{IMPLICATIONS}

We have found that the ultraviolet spectrum of PG 1159-035 is dominated by features due to highly-ionized carbon, nitrogen and oxygen. While the identified ions are consistent with the diffusion calculations reported by Vauclair (1989) assuming a helium-rich atmosphere, the result underscores the possibility that the atmosphere may be dominated by a combination of CNO elements. This would be consistent with the pulsational driving mechanism of Starrfield et al. (1984). A detailed analysis employing non-LTE atmospheres treating the CNO ions will clarify this question, and the paper of Werner, Heber and Hunger (1989) represents the first such exploratory calculations.

This work was supported in part by NASA IUE grant NAG 5-38, by NSF grant AST 88-40482 and by the NSERC Canada.

\section{REFERENCES}

Bond, H.E., Grauer, A.D., Green, R.F. and Liebert, J. (1984). Astrophys. J. 279, 751.

Husfeld, D. (1987). In The Second Conference on Faint Blue Stars, IAU Coll. 95, edited by A.G.D. Philip, D.S. Hayes and J. Liebert (Davis, Schenectady NY) p. 237.

Kudritzki, R. (1987). In The Second Conference on Faint Blue Stars, IAU Coll. 95, edited by A.

G. Davis Philip, D. S. Hayes and J. W. Liebert (Davis, Schenectady NY), p. 177.

McGraw, J.T., Starrfield, S.G., Liebert, J. and Green, R.F. (1979). In White Dwarfs and Variable

Degenerate Stars, Proc. IAU Coll. 53, edited by H.M. Van Horn and V. Weidemann, (Univ. of Rochester Press, Rochester NY), p. 377.

Sion, E.M., Liebert, J. and Starrfield, S.G. (1985). Astrophys. J. 292, 471.

Starrfield, S.G., Cox, A.N., Kidman, R.B. and Pesnell, W.D. (1984). Astrophys. J. 281, 800.

Vauclair, G. 1989, this conference.

Werner, K., Heber, U. and Hunger, K. 1989, this conference.

Wesemael, F., Green, R.F. and Liebert, J. (1985). Astrophys. J. Suppl. Ser. 58, 379 (WGL). 